\title{
Performance Analysis of A Threshold-Based Dynamic TXOP Scheme for Intra-AC QoS in Wireless LANs
}

\author{
Jia $\mathrm{Hu}^{1}$, G. $\mathrm{Min}^{2}$, M. E. Woodward ${ }^{2}$ \\ ${ }^{1}$ Department of Mathematics and Computer Science, Liverpool Hope University, L16 9JD, UK \\ ${ }^{2}$ Department of Computing, University of Bradford, Bradford, BD7 1DP, U.K. \\ E-mail: huj@hope.ac.uk, \{g.min, m.e.woodward\}@brad.ac.uk
}

\begin{abstract}
The IEEE 802.11e Enhanced Distributed Channel Access (EDCA) protocol has been proposed for provisioning of differentiated Quality-of-Service (QoS) between various Access Categories (ACs), i.e., inter-AC QoS, in Wireless Local Area Networks (WLANs). However, the EDCA lacks the support of the intra-AC QoS provisioning, which is indispensable in the practical WLANs since the network loads are always asymmetric between traffic flows of ACs with the same priority. To address the intra-AC QoS issue, this paper proposes a Threshold-Based Dynamic Transmission Opportunity (TBD-TXOP) scheme which sets the TXOP limits adaptive to the current status of the transmission queue based on the pre-setting threshold. An analytical model is further developed to evaluate the QoS performance of this scheme in terms of throughput, end-to-end delay, and frame loss probability. NS-2 simulation experiments validate the accuracy of the proposed analytical model. The performance results demonstrate the efficacy of TBD-TXOP for the intra-AC QoS differentiation.

Index Terms: Transmission Opportunity; EDCA; Intra-AC QoS
\end{abstract}

\section{INTRODUCTION}

Distributed Coordination Function (DCF) ratified in the IEEE 802.11 standard [8] is the popular Medium Access Control (MAC) protocol deployed in the Wireless Local Area Network (WLAN) devices. However, due to the lack of support for real-time services, DCF is unable to provide Quality-of-Service (QoS) required by multimedia applications. With the ever-increasing demand of various wireless services, 
the provisioning of differentiated QoS has become a critical issue of the future wireless multimedia communication.

In order to support multimedia applications subject to the differentiated QoS constraints, the IEEE 802.11e has been standardized [8]. It introduces a contention-based channel access protocol, referred to as the Enhanced Distributed Channel Access (EDCA). The EDCA provides service differentiation by classifying the traffic flows into four Access Categories (ACs) [8], each of which is associated to a separate transmission queue and behaves independently. These ACs are differentiated through adjusting the parameters of Arbitrary Inter-Frame Space (AIFS), Contention Window (CW), and Transmission Opportunity (TXOP) limit [8].

Although the EDCA provides the QoS differentiation between various ACs, i.e., inter-AC QoS, it lacks the support of the intra-AC QoS provisioning [11, 12, 15]. The EDCA assigns the same MAC parameters to the traffic flows belonging to the same service class regardless of their traffic arrival rates and QoS requirements, which leads to the throughput fairness among the flows in the same class. However, in the practical WLANs with multimedia applications, the network loads are always asymmetric between traffic flows of ACs with the same priority. Therefore, it is desirable to provide intra-AC QoS differentiation between those flows, which belong to the same service class but have different traffic arrival rates and QoS requirements.

We use the TXOP limit as a way of intra-AC QoS differentiation in this study. The default TXOP scheme assigns a fixed TXOP limit to all the flows of the same service class [8]. We propose that each flow in the same class can have a dynamic TXOP limit that depends on its traffic arrival rate. Bearing in mind that if a flow has a higher traffic rate than the others of the same class, the transmission queue length of this flow should be larger than the others, we take the queue length as an indicator for the traffic rate. It is thus desirable to dynamically adjust the TXOP limits according to the status of the transmission queue. 
In order to support intra-AC QoS differentiation in IEEE 802.11e networks, in this paper, we propose a Threshold-Based Dynamic TXOP (TBD-TXOP) scheme and further develop a new analytical model to evaluate this scheme in WLANs. The main contributions of this paper include

(1) A threshold-based dynamic TXOP scheme is proposed to address the intra-AC QoS differentiation in IEEE 802.11e networks. This scheme dynamically adjusts the TXOP limits according to the current status of the transmission queue and the pre-setting threshold.

(2) A new analytical model is developed to evaluate the performance of the TBD-TXOP scheme. To make this challenging problem tractable, we use a Markov chain to solve the bulk service queueing system arising from the burst transmission. NS-2 simulation experiments validate the accuracy of the proposed analytical model. The performance results demonstrate that the TBD-TXOP scheme can effectively support the intra-AC QoS differentiation.

The rest of this paper is organized as follows. Section II introduces the EDCA protocol. Section III entails the TBD-TXOP scheme. Section IV reviews the related work in the literature. The analytical model for the proposed scheme is developed in Section V. Section VI presents the validation and performance analysis. Finally, the paper is concluded in Section VII.

\section{ENHANCED Distributed ChANNEl ACCESS}

EDCA has been designed to support multimedia applications with stringent QoS requirements in WLANs [8]. Traffic of different classes in terms of voice, video, best-effort, and background is assigned to one of four ACs, which is associated to a separate transmission queue and behaves independently. The QoS of these ACs is differentiated through assigning various EDCA parameters including AIFS values, $\mathrm{CW}$ sizes, and TXOP limits. In the EDCA protocol, the channel is sensed before an AC attempts to transmit frames. If the channel is detected idle for an AIFS, the transmission starts. Otherwise, the AC defers until the channel is detected idle for an AIFS, and then generates a random backoff counter. The value of the backoff counter is uniformly chosen between zero and $C W$, which is initially set to $C W_{\text {min }}$ 
and doubled after each unsuccessful transmission until it reaches the maximum value $C W_{\max }$. It is reset to $C W_{\text {min }}$ after the transmission succeeds or the number of retransmission attempts reaches a retry limit. The backoff counter is decreased by one for each time slot [8] when the channel is idle, halted when the channel becomes busy and resumed when the channel is idle again for an AIFS. An AC transmits when its backoff counter becomes zero.

When an AC wins the contention for the channel, it transmits the frames available in its buffer successively provided that the duration of transmission does not exceed the specified TXOP limit [8]. Each frame is acknowledged by an Acknowledgement (ACK) after a Short Inter-frame Space (SIFS) interval. The next frame is transmitted immediately after receiving the ACK and waiting for an SIFS. If the transmission of any frame fails the burst is terminated and the AC contends again for the channel to retransmit the failed frame. The TXOP scheme is an efficient way to improve the network utilization because the backoff overhead is shared among all the frames transmitted within a burst.

\section{RELATED WORK}

A significant amount of work has been reported on the performance analysis of DCF [1, 2, 17, 20, 26] and EDCA [4-6, 7, 9, 11-13, 15, 16, 18, 19, 21-25]. Majority of the studies for EDCA were focused on the AIFS and CW schemes [4, 7, 9, 12, 19, 24]. For instance, Xiao [24] presented a bi-dimensional Markov chain model for the CW differentiation scheme. Huang and Liao [7] analyzed the performance of saturation throughput and access delay of EDCA with AIFS and CW differentiation. Ramaiyan, Kumar, and Altman [19] proposed the fixed point analysis to capture AIFS and $\mathrm{CW}$ differentiation and established a condition for the uniqueness of the fixed point solution. Gas et al. [4] presented a 3D EDCA model implementing CW and AIFS built on an existing comprehensive model in [5].

As the TXOP scheme can not only support service differentiation but also improve the network utilization, performance evaluation of this scheme has also received many research efforts recently [5, 6 , 
11, 13-16, 18, 21, 22, 25]. Most existing analytical models for TXOP have been developed under the assumption of saturated traffic loads and thus excluded any need to consider queueing or traffic models for performance analysis [13, 18, 22, 25]. For instance, Tinnirello and Choi [22] compared the saturation throughput of the TXOP scheme coupled by different ACK policies. Li, Ni, and Xiao [13] analyzed the TXOP scheme with the block ACK policy under saturated traffic loads and noisy channel conditions. Xu, Sakurai, and $\mathrm{Vu}[25]$ proposed an access delay model for EDCA with the AIFS, CW, and TXOP schemes under saturated conditions. Since the realistic network conditions are often unsaturated, it is important to evaluate the performance of the TXOP scheme under unsaturated traffic loads $[5,6,14,16,21]$. Tickoo and Sikdar [21] used a discrete-time G/G/1 queueing system with infinite buffer capacity to model the DCF and extended the queueing model to analyze the TXOP scheme under unsaturated conditions. Hu et al. $[5,6]$ developed a comprehensive EDCA model with the combination of AIFS, CW, and TXOP schemes under unsaturated traffic loads.

The aforementioned work on EDCA was primarily focused on the QoS differentiation between various ACs, namely, inter-AC QoS. However, since the network loads are always asymmetric between traffic flows in the practical WLANs, it is desirable to provide the intra-AC QoS between the traffic flows of ACs with the same priority, regarding to their traffic arrival rates and QoS constraints. To tackle the intra-AC QoS issue, several TXOP-based approaches $[11,12,15]$ have been reported in the literature. Ksentini et al. [11] proposed an enhanced TXOP scheme where each traffic flow monitors the MAC queue and computes at runtime the TXOP limit according to the flow's AC and the queue length. However, the TXOP limits for the ACs with video traffic is set to the number of arriving frames between two successful transmission attempts, which is obtained through simulations [11]. Liu and Zhao [15] introduced a new TXOP scheme, which takes into account the frame size and the transmission queue length to tune the TXOP limit to improve the QoS support for VBR video transmission over the IEEE 802.11e WLANs. However, the performance of this scheme [15] was evaluated through simulations and no theoretical analysis was given. Kosek-Szott [12] develops an analytical model to evaluate the throughput performance 
for IEEE 802.11aa intra-access category prioritization including virtual collision handling, backoff differentiation, and Arbitration Inter-Frame Space differentiation. Distinguished from the existing solutions for the intra-AC QoS issue, we propose a threshold-based TXOP scheme that dynamically adjusts the TXOP limits according to the current status of the transmission queue and the pre-setting threshold. This model can be used to derive the QoS performance metrics in terms of throughput, end-to-end delay, and frame loss probability. The efficacy of this scheme is verified through both the analytical results and simulation experiments.

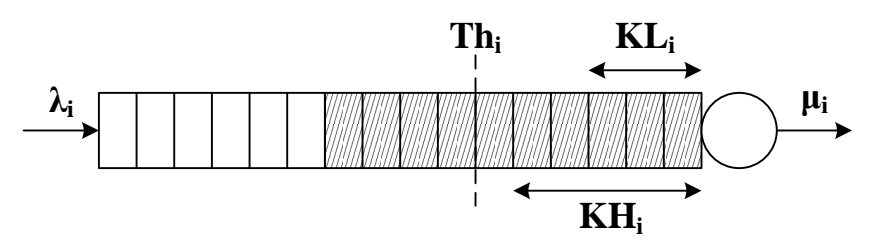

Fig. 1. A queue instance for the TBD-TXOP scheme

\section{TBD-TXOP}

In the original TXOP scheme, the TXOP limits for each AC are fixed [8]. In order to provide the intra-AC QoS, we propose to dynamically adjust the TXOP limit according to the status of the current transmission queue. Specifically, we adopt two TXOP limits including a low and a high value for the ACs of the real-time traffic in terms of voice and video. For the ACs of the best-effort and background traffic, the TXOP limits are equal to one (frame) as in the EDCA protocol [8]. Particularly, suppose the TXOP limits for the ACs from the lowest to the highest priority are fixed at $K_{0}, K_{1}, K_{2}, K_{3}\left(K_{0} \leq K_{1} \leq K_{2} \leq K_{3}\right)$ in the EDCA protocol, respectively. Then in the TBD-TXOP Scheme, the TXOP limits for the ACs from the lowest to the highest priority are set to $\left(1, K_{0}\right),\left(K_{0}, K_{1}\right),\left(K_{1}, K_{2}\right),\left(K_{2}, K_{3}\right)$, respectively, where the $\mathrm{AC}$ of each priority has two TXOP limits: a low and a high TXOP. The switch between the low and high value is triggered by a threshold that is used to differentiate the QoS of the traffic flows of ACs with the same priority. As shown in Fig. 1, when the queue length is below a certain threshold, the TXOP limit is fixed at the low value. However, if the 
queue length equals or exceeds the threshold, the TXOP limit is augmented to the high value. In what follows, the low TXOP value, high TXOP value, and threshold are denoted as $K L_{i}, K H_{i}$, and $T h_{i}$, respectively, with the subscript $i$ representing the identity number of the traffic flow.

TABLE I

SUMMARY OF THE MAIN NOTATIONS

\begin{tabular}{|c|c|}
\hline$n$ & number of ACs \\
\hline$\lambda_{i}$ & mean traffic arrival rate of the $\mathrm{AC}_{\mathrm{i}}$ \\
\hline$p_{i}$ & collision probability of the $\mathrm{AC}_{\mathrm{i}}$ \\
\hline$\tau_{i}$ & transmission probability of the $\mathrm{AC}_{\mathrm{i}}$ \\
\hline$\pi_{0 i}$ & probability that the transmission queue of the $\mathrm{AC}_{\mathrm{i}}$ being empty \\
\hline$W_{i}$ & minimum contention window of the $\mathrm{AC}_{\mathrm{i}}$ \\
\hline$m_{i}$ & maximum backoff stage \\
\hline$E\left[S_{v i}\right]$ & mean service time of the $\mathrm{AC}_{\mathrm{i}}$ with $v$ frames in the burst \\
\hline$E\left[A_{i}\right]$ & mean channel access delay of the $\mathrm{AC}_{\mathrm{i}}$ \\
\hline$E\left[B_{v}\right]$ & mean burst transmission time of a burst with $v$ frames \\
\hline $\bar{\sigma}_{i}$ & average length of a time slot perceived by the $\mathrm{AC}_{\mathrm{i}}$ \\
\hline$\sigma$ & length of a physical time slot specified in the 802.11 standard \\
\hline$P T_{i}$ & $\begin{array}{l}\text { probability that at least one of the remaining } \mathrm{ACs} \text { transmits given the } \mathrm{AC}_{\mathrm{i}} \text { being in the backoff } \\
\text { procedure }\end{array}$ \\
\hline$P S_{i j}$ & $\begin{array}{l}\text { probability that the } \mathrm{AC}_{\mathrm{j}} \text { successfully transmits among the remaining } \mathrm{ACs} \text { given the } \mathrm{AC}_{\mathrm{i}} \text { being } \\
\text { in the backoff procedure }\end{array}$ \\
\hline$T_{c}$ & average collision time \\
\hline$T_{s}^{j}$ & average time of the successful transmission of a burst with $s$ frames from the $\mathrm{AC}_{\mathrm{j}}$ \\
\hline$K H_{i}$ & high TXOP value \\
\hline$K L_{i}$ & low TXOP value \\
\hline$T h_{i}$ & threshold of the switch between the high and low TXOP values \\
\hline$L_{v j}$ & probability that there are $v$ frames transmitted in the burst from the $\mathrm{AC}_{\mathrm{j}}$ \\
\hline$\mu_{v i}$ & mean service rate of the the $\mathrm{AC}_{\mathrm{i}}$ with $v$ frames in the burst \\
\hline$N$ & buffer size at each AC \\
\hline G & transition rate matrix of the Markov chain of the $\mathrm{AC}_{\mathrm{i}}$ shown in Fig. 2 \\
\hline $\mathbf{P}$ & steady-state probability vector of the Markov chain of the $\mathrm{AC}_{\mathrm{i}}$ shown in Fig. 2 \\
\hline$\Gamma_{i}$ & throughput of the $\mathrm{AC}_{\mathrm{i}}$ \\
\hline Loss $_{i}$ & frame loss probability of the $\mathrm{AC}_{\mathrm{i}}$ \\
\hline$E\left[D_{i}\right]$ & total frame delay of the $\mathrm{AC}_{\mathrm{i}}$ \\
\hline
\end{tabular}




\section{The ANALYTICAL MODEL FOR THE TBD-TXOP SCHEME}

In this section, we develop an analytical model for the proposed TBD-TXOP scheme in IEEE 802.11e WLANs. Since we focus on the intra-AC QoS issue, each station only has traffic belonging to a single AC and all the ACs are of the same priority. Without loss of generality, we consider a scenario of $n$ ACs where the mean traffic arrival rate of $A C_{i}(i=1,2, \cdots, n)$ is $\lambda_{i}$. The transmission queue of each $\mathrm{AC}$ is modelled as a bulk service queueing system to capture the burst transmission property of the TBD-TXOP scheme. The service time of the queueing system can be obtained by analyzing the backoff and burst transmission procedures. For clarity of the derivation of the analytical model, Table I lists the main notations used in this paper.

\section{A. Analysis of the Backoff and Burst transmission Procedures}

Only the Head-of Burst (HoB) frame needs to contend for the channel. After winning the contention for the channel, the whole burst can be transmitted successfully given the wireless channel is error-free. Therefore, the "transmission probability" and "collision probability" mean the "transmission probability of an $\mathrm{HoB}$ frame" and the "collision probability of an $\mathrm{HoB}$ frame" in the following. The transmission probability can be approximated by weighting the saturated transmission probability with the probability of a non-empty buffer [21,26]. Therefore, the transmission probability of $A C_{i}, \tau_{i}$, is given by [2]

$$
\tau_{i}=\frac{2\left(1-2 p_{i}\right)\left(1-\pi_{0 i}\right)}{\left(1-2 p_{i}\right)\left(W_{i}+1\right)+p_{i} W_{i}\left(1-\left(2 p_{i}\right)^{m_{i}}\right)}
$$

where $W_{i}$ is the minimum contention window and $m_{i}$ represents the maximum backoff stage. $\pi_{0 i}$ denotes the probability that the transmission queue of $A C_{i}$ is empty.

The collision probability experienced by a transmitted $\mathrm{HoB}$ frame, $p_{i}$, is the probability that at least one of the remaining ACs transmits in the considered time slot. Thus, $p_{i}$ is given by

$$
p_{i}=1-\prod_{i=1}^{n}\left(1-\tau_{i}\right)
$$

We define the service time as the duration from the instant that an $\mathrm{HoB}$ frame starts contending for the channel to the instant that the burst is acknowledged following successful transmission. The service 
time includes two parts: the channel access delay and burst transmission delay. The former is the time duration from the instant that the frame reaches the head of its transmission queue until it wins the contention and is ready for transmission. The latter is the time duration of successfully transmitting a burst. Let $E\left[S_{v i}\right], E\left[A_{i}\right]$ and $E\left[B_{v}\right]$ denote the mean of the service time, channel access delay and burst transmission delay, respectively, where $v$ represents the number of frames transmitted within a burst and $i$ denotes that the burst transmission is from the $A C_{i} . E\left[A_{i}\right]$ can be calculated as

$$
E\left[A_{i}\right]=T_{c} \frac{p_{i}}{1-p_{i}}+\bar{\sigma}_{i} \sum_{s=0}^{\infty} \sum_{h=0}^{s} \frac{W_{h i}-1}{2} p_{i}^{s}\left(1-p_{i}\right)
$$

where $T_{c}$ is the average collision time and $\bar{\sigma}_{i}$ is the average length of a time slot perceived by the $A C_{i} \cdot p_{i}^{s}\left(1-p_{i}\right)$ is the probability that the HoB frame is successfully transmitted after $s$ collisions, and $\left(W_{h i}-1\right) / 2$ is the average value of the backoff counters generated in the $h$-th backoff stage.

Let $P T_{i}$ represent the probability that at least one of the remaining ACs transmits in a given time slot when the $A C_{i}$ is in the backoff state. $P T_{i}$ is equal to the collision probability, $p_{i}$, when the $A C_{i}$ transmits, as given in Eq. (2). Let $P S_{i j}$ denote the probability that there is a successful transmission from the $A C_{j}$ when the $A C_{i}$ is in the backoff state. $P S_{i j}$ can be given by

$$
P S_{i j}=\tau_{j} \prod_{r \neq i, j}\left(1-\tau_{r}\right)
$$

The average length of a time slot, $\bar{\sigma}_{i}$, is obtained by considering the fact that the channel is idle with probability $\left(1-P T_{i}\right)$, a successful transmission occurs with probability $\sum_{j=1}^{n} P S_{i j}$, and a collision happens with probability $\left(P T_{i}-\sum_{j=1}^{n} P S_{i j}\right)$.

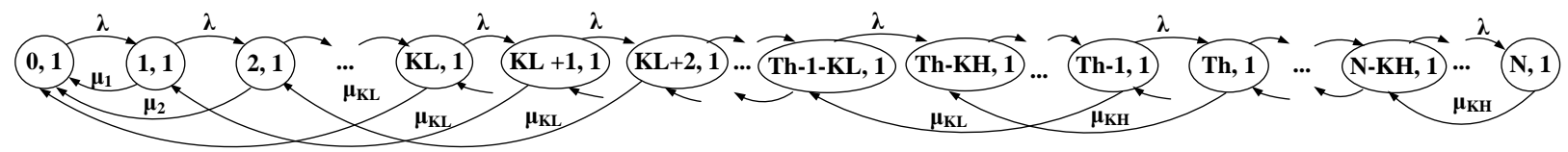

Fig. 2. The state-transition-rate diagram of the $\mathbf{M} / \mathbf{G}^{\left[1, K H_{i}\right]} / \mathbf{1} / \mathbf{N}$ queuing system. (the subscript $i$ is removed for clarity of the figure)

$$
\bar{\sigma}_{i}=\left(1-P T_{i}\right) \sigma+\sum_{j=1}^{n} P S_{i j} T_{j}+\left(P T_{i}-\sum_{j=1}^{n} P S_{i j}\right) T_{c}
$$


where $\sigma$ is the duration of a physical time slot and $T_{s}^{j}$ is the average time for the successful transmission of a burst from the $A C_{j}$.

Note that only the HoB frame can be involved in a collision. $T_{c}$ is given by

$$
T_{c}=T_{A I F S}+T_{L}+T_{H}+T_{S I F S}+T_{A C K}
$$

where $T_{L}$ and $T_{H}$ are the average time required for transmitting the frame payload and the frame header, respectively. The average time for the successful transmission of a burst, $T_{s}^{j}$, can be expressed as

$$
T_{s}^{j}=\frac{\sum_{v=1}^{K L_{j}} E\left[B_{v}\right] L_{v j}+E\left[B_{K H_{j}}\right] L_{K H_{j}}}{\left(1-\pi_{0 j}\right)}
$$

where $K L_{j}$ and $K H_{j}$ denote the maximum number of frames that can be transmitted in the low and the high TXOP limits, respectively, the denominator $\left(1-\pi_{0 j}\right)$ means that the occurrence of burst transmission is conditioned on the fact that there is at least one frame in the transmission queue, and $L_{v j}$ $\left(1 \leq v \leq K L_{j}\right.$ or $\left.v=K H_{j}\right)$ is the probability that $v$ frames are transmitting form the $A C_{j}$ within a TXOP. $E\left[B_{v}\right]$ is the burst transmission delay that is dependent on the number of frames transmitted within a burst and can be given by

$$
E\left[B_{v}\right]=T_{A I F S}+v\left(T_{L}+T_{H}+2 T_{S I F S}+T_{A C K}\right)-T_{S I F S}
$$

\section{B. Queueing Analysis}

The transmission queue at the $A C_{i}$ with Poisson arrival process can be characterized as an $\mathrm{M} / \mathrm{G}^{\left[1, K H_{i}\right]} / 1 / \mathrm{N}$ queueing system [20], where the superscript $\left[1, K H_{i}\right]$ denotes that the number of frames transmitted in a burst from the $A C_{i}$ ranges from 1 to $K H_{i}$, and $N$ represents the buffer size at each AC. The service time of the queueing system can be modelled by an exponential distribution function with mean $E\left[S_{v i}\right]$. Thus, the service rate, $\mu_{v i}$, is given by $1 / E\left[S_{v i}\right]$.

The queueing system of the $A C_{i}$ can be modelled by a Markov chain with $(N+1)$ states where state $k, \quad(k=0,1, \ldots, N)$ denotes that there are $k$ frames in the system. Fig. 2 illustrates the Markov chain. The transition rate out of state $k$ to $(k+1)$ is $\lambda_{i}$, which is the traffic arrival rate. A transition 
from state $k$ to $\left(k-K H_{i}\right),\left(T h_{i} \leq k \leq N\right)$, is $\mu_{K H_{i}}$, from state $k$ to $\left(k-K L_{i}\right), \quad\left(K L_{i} \leq k<T h_{i}\right)$, is $\mu_{K L_{i}}$, and from state $k$ to $0,\left(1 \leq k \leq K L_{i}-1\right)$, is $\mu_{k i}$.

We can obtain the transition rate matrix, G, of the bi-variate Markov chain from the state-transition-rate diagram. The steady-state probability vector, $\mathbf{P}=\left(P_{k}\right)=\left(P_{0}, P_{1}, \cdots, P_{N}\right)$ where $\mathbf{P}$ satisfies the following equations:

$$
\mathbf{P G}=0 \text { and } \mathbf{P e}=1
$$

where $\mathbf{e}$ is a unit column vector of the size $(N+1)$. After obtaining $\mathbf{P}$, we can express $L_{v i}$, the probability that $v$ frames are transmitting form the $A C_{i}$ within a TXOP as

$$
L_{v i}= \begin{cases}P_{v}, & 1 \leq v<K L_{i}, \\ \sum_{z=K L_{i}}^{T h_{i}-1} P_{\mathrm{z}}, & v=K L_{i} \\ \sum_{z=T H_{i}}^{N} P_{\mathrm{z}}, & v=K H_{i} .\end{cases}
$$

Let $\pi_{k i}$ denote the steady-state probability that there are $k$ frames in the queueing system of the $A C_{i} . \pi_{k i}$ is given by

$$
\pi_{k i}=\mathbf{P e}, \quad 0 \leq k \leq N
$$

Thus we can have the probability, $\pi_{0 i}$, that the transmission queue is empty.

Therefore, the frame loss probability, Loss $_{i}$, that an arriving frame finds the finite buffer of the $A C_{i}$ full, is given by $\pi_{N i}$. Given the loss probability $\operatorname{Loss}_{i}$, the throughput $\Gamma_{i}$ of the $A C_{i}$ can be computed by

$$
\Gamma_{i}=\lambda_{i} E[P]\left(1-\operatorname{Loss}_{i}\right)
$$

where $E[P]$ is the frame payload length and $\lambda_{i}$ is the mean traffic arrival rate of the $A C_{i}$.

The end-to-end delay, which equals to the queueing delay plus service time, is the time duration from the instant that a frame enters the transmission queue of the $\mathrm{AC}$, to the instant that the frame is acknowledged after successful transmission. By virtue of Little's Law [20], The end-to-end delay, $E\left[D_{i}\right]$, is given by 


$$
E\left[D_{i}\right]=\frac{E\left[N_{i}\right]}{\lambda_{i}\left(1-\text { Loss }_{i}\right)}
$$

where $E\left[N_{i}\right]=\sum_{k=1}^{N} k \pi_{k i}$ is the average number of frames in the queueing system of the $A C_{i}$. $\lambda_{i}\left(1-\operatorname{Loss}_{i}\right)$ is the effective arrival rate to the transmission queue of the $A C_{i}$ since the arriving frames are dropped if the finite buffer becomes full.

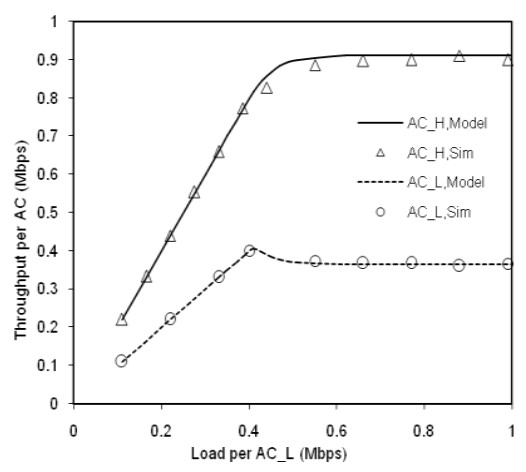

(a) Throughput

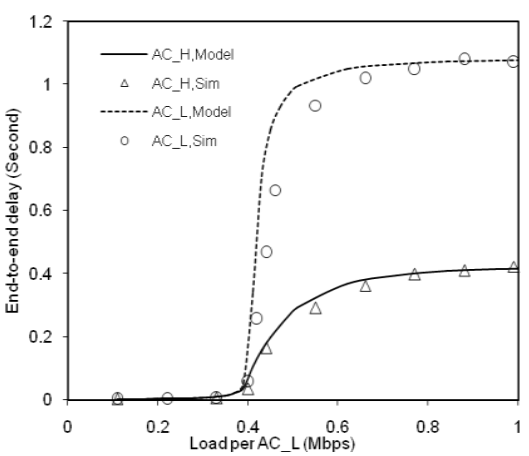

(b) End-to-end delay

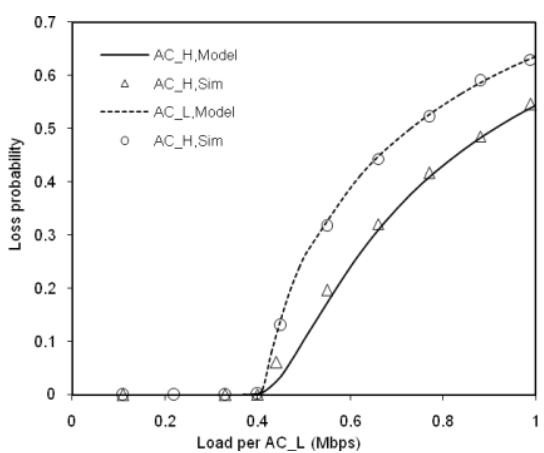

(c) Frame loss probability

Fig. 3. Validation results of the analytical model

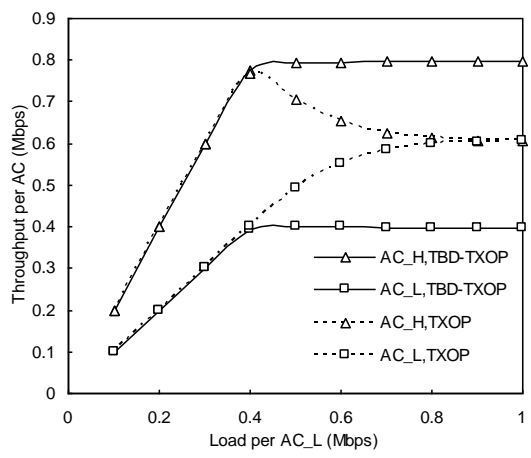

(a) Throughput

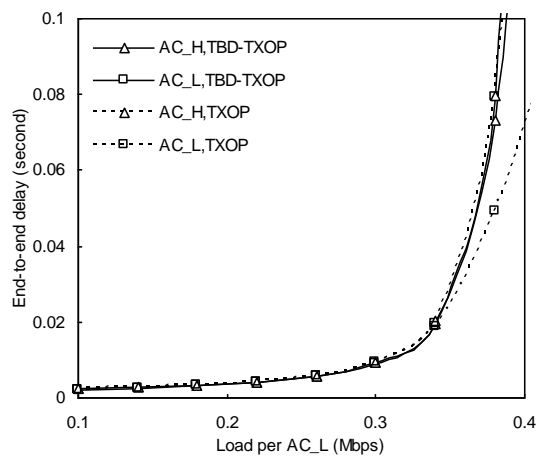

(b) End-to-end delay

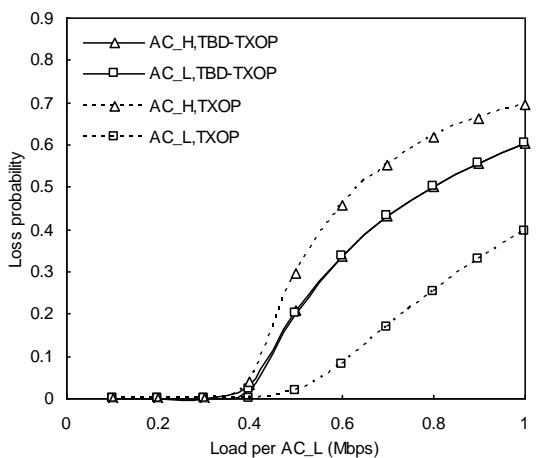

(c) Frame loss probability

Fig. 4. Analytical results of the TBD-TXOP and TXOP schemes

TABLE II

SYSTEM PARAMETERS

\begin{tabular}{|l|l|l|l|}
\hline $\begin{array}{l}\text { Frame } \\
\text { payload }\end{array}$ & 8000 bits & PHY header & 192 bits \\
\hline MAC header & 224 bits & ACK & 112 bits + PHY header \\
\hline Channel rate & $11 \mathrm{Mbps}$ & $\mathrm{CW}_{\min }$ & 32 \\
\hline Basic rate & $1 \mathrm{Mbps}$ & $\mathrm{CW}_{\max }$ & 1024 \\
\hline Slot time & $20 \mu \mathrm{s}$ & Buffer size & 50 frames \\
\hline SIFS & $10 \mu \mathrm{s}$ & AIFS & $50 \mu \mathrm{s}$ \\
\hline
\end{tabular}




\section{Performance Analysis}

This section uses the model to investigate the performance of the TBD-TXOP scheme. We consider a WLAN where all the stations are located in a rectangular grid with dimension $100 \mathrm{~m} \times 100 \mathrm{~m}$ and are within the carrier sensing and transmission range of each other. Each station has traffic belonging to a single AC and all the ACs are of the same priority. There are two different types of traffic flows where the ones with the high and the low traffic arrival rates are denoted as "AC_H" and "AC_L", respectively. The traffic arrival rates at the "AC_H" flows are set to be double of those at the "AC_L" flows. The traffic generated by each AC follows the Poisson arrival process. Each simulation is executed once with 600 seconds simulation time, which is sufficiently long as the simulation results do not change with any further increment of simulation time. The two-ray ground propagation model is used. The system parameters used in this study follow the IEEE 802.11b standard [8] using Direct Sequence Spread Spectrum (DSSS) as the physical-layer technology, and are summarized in Table II.

Fig. 3 plots the results of the throughput, end-to-end delay, frame loss probability versus the traffic loads of the "AC_L" flows, respectively. There are 10 stations where 4 generates "AC_H" traffic flows and the rest generates "AC_L" flows. The low and the high TXOP limits are set to $K L=2$ and $K H=5$ (frames), respectively. The thresholds in the TBD-TXOP scheme are 3 and 50 frames for "AC_H" and "AC_L" traffic flows, respectively. As shown in Fig. 3, the good degree of agreement between the analytical results and the simulation experiments demonstrates the accuracy of the model for evaluating the QoS performance of the TBD-TXOP scheme.

In Fig. 4, we compare the throughput, end-to-end delay, and frame loss probability of the ACs with the TBD-TXOP scheme to those with the TXOP scheme. There are 10 stations where half generates "AC_H" traffic flows and the other half generates "AC_L" flows. The low and the high TXOP limits are

set to $K L=3$ and $K H=6$ (frames), respectively. The thresholds in the TBD-TXOP scheme are 4 and 50 frames for "AC_H" and "AC_L" traffic flows, respectively, which means that we set the largest degree of differentiation between these two types of flows that can be achieved using the TBD-TXOP scheme. 
Fig. 4 clearly demonstrates that the TBD-TXOP scheme is capable of providing intra-AC QoS differentiation. Particularly, the throughput, end-to-end delay, and frame loss probability of the "AC_H" flows improve while those of the "AC_L" flows deteriorate with the TBD-TXOP scheme.

\section{CONCLUSIONS}

In order to support the intra-AC QoS differentiation in IEEE 802.11e networks, a dynamic TXOP scheme named TBD-TXOP has been proposed, which sets the TXOP limits adaptive to the current status of the transmission queue based on the pre-setting threshold. With the aim of investigating the performance of the TBD-TXOP scheme, an analytical model has been developed for this scheme in WLANs with unbalanced traffic flows. QoS performance metrics in terms of throughput, end-to-end delay, and frame loss probability have been derived. The accuracy of the analytical model has been validated by NS-2 simulation results. Moreover, the performance results have demonstrated the efficacy of TBD-TXOP for intra-AC QoS differentiation between unbalanced traffic flows in IEEE 802.11e WLANs. Our possible future work could be to apply and change the proposed model to investigate the intra-AC problem in 802.11aa, and the TBD-TXOP scheme could be further extended and improved to adapt to the practical multimedia traffic including video and voice.

\section{REFERENCE}

[1] A. Abdrabou and W. Zhuang, "Service Time Approximation in IEEE 802.11 Single-Hop Ad Hoc Networks," IEEE Transactions on Wireless Communications, vol. 7, no. 1, pp. 305-313, 2008.

[2] G. Bianchi, "Performance Analysis of the IEEE 802.11 Distributed Coordination Function," IEEE Journal on Selected Areas in Communications, vol. 18, no. 3, pp. 535-547, 2000.

[3] W. Fischer and K. Meier-Hellstern, "The Markov-Modulated Poisson Process (MMPP) Cookbook," Performance Evaluation, vol. 18, no. 2, pp. 149-171, 1993.

[4] M. Gas, K. Kosek-Szott, M. Natkaniec, A. R. Pach, "3D Markov chain-based saturation throughput model of IEEE 802.11 EDCA," Electronics Letters, vol. 47, no. 14, 2011. 
[5] J. Hu, G. Min, M. E. Woodward, and W. Jia, "A Comprehensive Analytical Model for IEEE 802.11e QoS Differentiation Schemes under Unsaturated Traffic Loads," Proc. IEEE ICC’08, pp. 241-245, 2008.

[6] J. Hu, G. Min, M. E. Woodward, and W. Jia, "Comprehensive QoS Analysis of Enhanced Distributed Channel Access in Wireless Local Area Networks," Information. Sciences, vol. 214, pp. 20-34, 2012.

[7] C. L. Huang and W. Liao, "Throughput and Delay Performance of IEEE 802.11e Enhanced Distributed Channel Access (EDCA) under Saturation Condition," IEEE Transactions on Wireless Communications, vol. 6, no. 1, pp. 136-145, 2007.

[8] IEEE, "Wireless LAN Medium Access Control (MAC) and Physical Layer (PHY) specifications: Medium Access Control (MAC) Quality of Service (QoS) Enhancements," IEEE Standard 802.11e, 2005 .

[9] S. Kim, R. Huang, and Y. Fang, "Deterministic Priority Channel Access Scheme for QoS Support in IEEE 802.11e Wireless LANs," IEEE Transactions on Vehicular Technology, vol. 58, no. 2, pp. $855-864,2009$.

[10] L. Kleinrock, Queueing Systems: Theory: John Wiley \& Sons, 1975.

[11] A. Ksentini, A. Nafaa, A. Gueroui, and M. Naimi, "ETXOP: A Resource Allocation Protocol for QoS-Sensitive Services Provisioning in 802.11 Networks," Performance Evaluation, vol. 64, no. 5, pp. 419-443, 2007.

[12] K. Kosek-Szott, "A Throughput Model of IEEE 802.11aa Intra-Access Category Prioritization," Wireless Personal Communications, available online. DOI: 10.1007/s11277-012-0861-6, 2012.

[13] T. Li, Q. Ni, and Y. Xiao, "Investigation of the Block ACK Scheme in Wireless Ad-Hoc Networks," Wireless Communications and Mobile Computing, vol. 6, no. 6, pp. 877-888, 2006.

[14] T. Li, D. Leith, V. Badarla, D. Malone, and Q. Cao, "Achieving End-to-end Fairness in 802.11e Based Wireless Multi-Hop Mesh Networks without Coordination", Mobile Networks and Applications, vol. 16, no.1, pp. 17-34, 2011.

[15] H. Liu and Y. Zhao, "Adaptive EDCA Algorithm Using Video Prediction for Multimedia IEEE 802.11e WLAN," Proc. IEEE ICWMC'06, pp. 10-15, 2006.

[16] K. Lu, D. Wu, Y. Qian, Y. Fang, and R. C. Qiu, "Performance of an Aggregation-based MAC Protocol for High Data Rate Ultra-Wideband Ad Hoc Networks," IEEE Transactions on Vehicular Technology, vol. 56, no. 1, pp. 312-321, 2007. 
[17] D. Malone, K. Duffy, and D. J. Leith, "Modeling the 802.11 Distributed Coordination Function in Non-saturated Heterogeneous Conditions," IEEE/ACM Transactions on Networking, vol. 15, no. 1, pp. 159-172, 2007.

[18] F. Peng, H. M. Alnuweiri, and V. C. M. Leung, "Analysis of Burst Transmission in IEEE 802.11e Wireless LANs," Proc. IEEE ICC'06, vol. 2, pp. 535-539, 2006.

[19] V. Ramaiyan, A. Kumar, and E. Altman, "Fixed Point Analysis of Single Cell IEEE 802.11e WLANs: Uniqueness and Multistability," IEEE/ACM Transactions on Networking, vol. 16, no. 5, pp. 1080-1093, 2008.

[20] G. Sharma, A. Ganesh, and P. Key, "Performance Analysis of Contention Based Medium Access Control Protocols," IEEE Transactions on Information Theory, vol. 55, no. 4, pp. 1665-1682, 2009.

[21] O. Tickoo and B. Sikdar, "Modeling Queueing and Channel Access Delay in Unsaturated IEEE 802.11 Random Access MAC Based Wireless Networks," IEEE/ACM Transactions on Networking, vol. 16, no. 4, pp. 878-891, 2008.

[22] I. Tinnirello and S. Choi, "Efficiency Analysis of Burst Transmission with Block ACK in Contention-based 802.11e WLANs," Proc. IEEE ICC'05, vol. 5, pp. 3455-3460, 2005.

[23] S. Tursunova and Y. T. Kim, "Realistic IEEE 802.11e EDCA model for QoS-Aware Mobile Cloud Service Provisioning” IEEE Transactions on Consumer Electronics, vol. 58, no. 1, pp. 60-68, 2012.

[24] Y. Xiao, "Performance Analysis of Priority Schemes for IEEE 802.11 and IEEE 802.11e Wireless LANs," IEEE Transactions on Wireless Communications, vol. 4, no. 4, pp. 1506-1515, 2005.

[25] D. Xu, T. Sakurai, and H. L. Vu, "An Access Delay Model for IEEE 802.11e EDCA," IEEE Transactions on Mobile Computing, vol. 8, no. 2, pp. 261-275, 2009.

[26] Q. Zhao, D. H. K. Tsang, and T. Sakurai, "A Simple and Approximate Model for Nonsaturated IEEE 802.11 DCF," IEEE Transactions on Mobile Computing, vol. 8, no. 11, pp. 1539-1553, 2009. 